\title{
PLEIADES ABSOLUTE CALIBRATION : INFLIGHT CALIBRATION SITES AND METHODOLOGY
}

\author{
S. Lachérade, S. Fourest, P. Gamet, L. Lebègue
}

CNES, French National Space Agency, 18 avenue Edouard Belin, 31401 Toulouse Cedex, France Phone: 33.(0)5.61.27.45.46 Fax: 33.(0)5.61.27.31.67 E-mail: sophie.lacherade@cnes.fr

ISPRS and IAA : Pléiades Inflight Calibration and Performance Assessment

KEY WORDS: Absolute Calibration, natural sites, calibration methods

\begin{abstract}
:
In-flight calibration of space sensors once in orbit is a decisive step to be able to fulfil the mission objectives. This article presents the methods of the in-flight absolute calibration processed during the commissioning phase. Four In-flight calibration methods are used: absolute calibration, cross-calibration with reference sensors such as PARASOL or MERIS, multi-temporal monitoring and inter-bands calibration. These algorithms are based on acquisitions over natural targets such as African deserts, Antarctic sites, La Crau (Automatic calibration station) and Oceans (Calibration over molecular scattering) or also new extra-terrestrial sites such as the Moon and selected stars. After an overview of the instrument and a description of the calibration sites, it is pointed out how each method is able to address one or several aspects of the calibration. We focus on how these methods complete each other in their operational use, and how they help building a coherent set of information that addresses all aspects of in-orbit calibration. Finally, we present the perspectives that the high level of agility of PLEIADES offers for the improvement of its calibration and a better characterization of the calibration sites.
\end{abstract}

\section{INTRODUCTION}

PLEIADES is a dual Earth observation system with a metric resolution, launched at the end of 2011. This imagery system, led by the French National Space Agency, CNES is derived from the instrument developed for the SPOT programs. It is composed of four spectral bands, blue, green, red and near infrared, with a resolution of $2.8 \mathrm{~m}$ in vertical viewing and a panchromatic band with a resolution of $0.7 \mathrm{~m}$ in vertical viewing (Figure 1). Its swath is about $20 \mathrm{~km}$. The targeted acquisition capabilities with high resolution and high repetitivity make it a particularly well adapted system for defense or civil security missions, as well as for critical geophysical phenomena surveying such as volcanic eruptions. Moreover, the spatial resolutions make it a system adapted to detailed mapping, especially in urban areas, that can be used to complement aerial photography. This system, launched at the end of 2011, should also complete the information supplied by other sensors, such as SPOT 5, for applications such as forestry, geology and marine environment.

This article describes the activities conducted during the commissioning phase of PLEIADES about the absolute radiometric calibration. The absolute coefficients are evaluated on ground before the launch but it is very important to confirm these values during the in-orbit life of the sensor and to follow the evolution of these coefficients if needed. The calibration phase could be done after the launch only once the images are well equalized. This means: after correction of the dark signals and after the correction of the non-uniformity of the detectors (Blanchet, 2012).

The methods which are applied must fulfill several specifications: an absolute specification for each spectral band, a multi-temporal specification to ensure the consistency of the data along the lifetime of the sensor and an interband specification to allow the users to derive spectral behaviours from PLEIADES images.

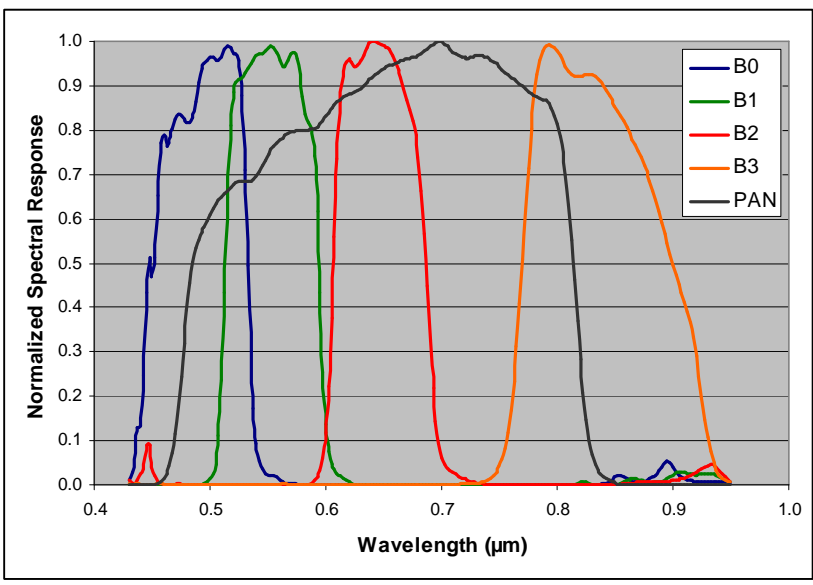

Figure 1. The PLEIADES.spectral bands.

First, this article describes the natural sites which are used for the PLEIADES absolute calibration. The second part of this article focuses on the methodology used during this commissioning phase. Then, the last part of this paper highlights new methods which will be developed through the PLEIADES programs to improve the calibration results taking into account the very high agility of the system.

\section{THE CALIBRATION SITES USED FOR PLEIADES}

Five types of natural sites are used for the absolute calibration: African deserts, snowy sites located in Antarctic, oceans, the La Crau site located in south East of France and the Moon. 


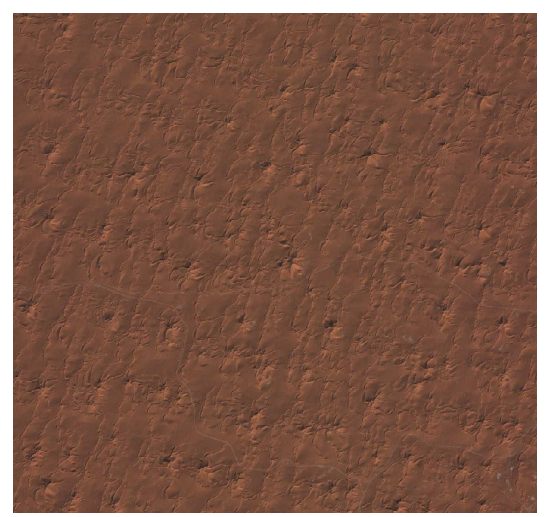

Algeria_3

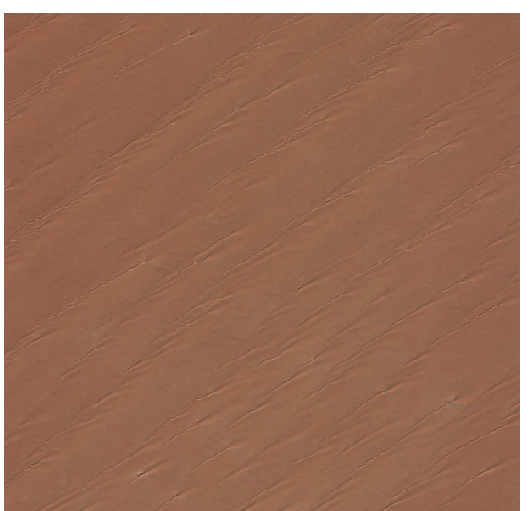

Libya_1

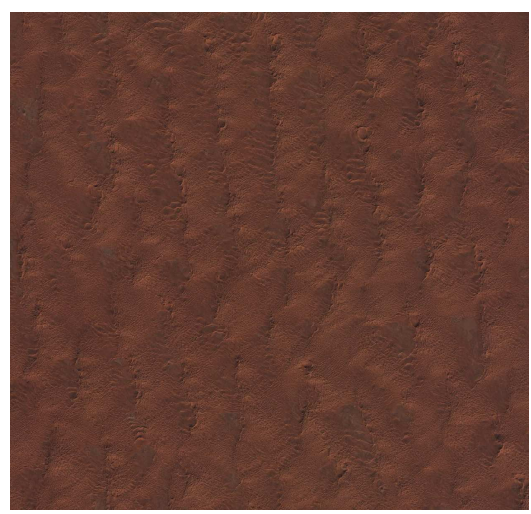

Algeria_5

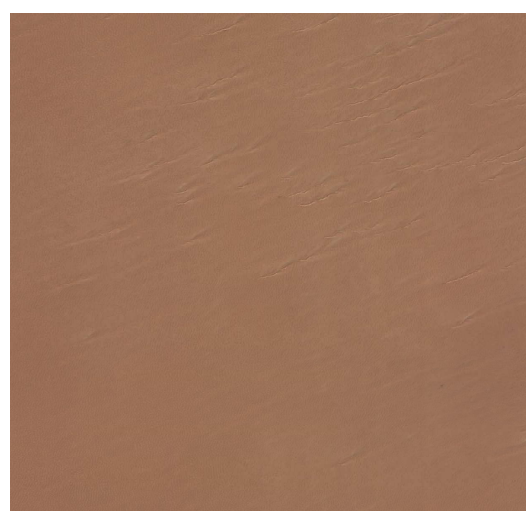

Mali_1

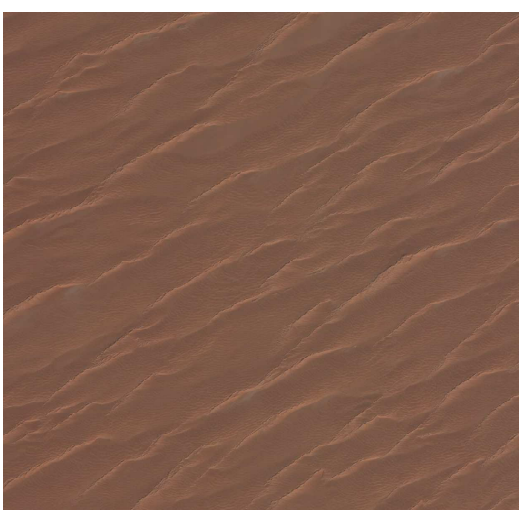

Arabia_1

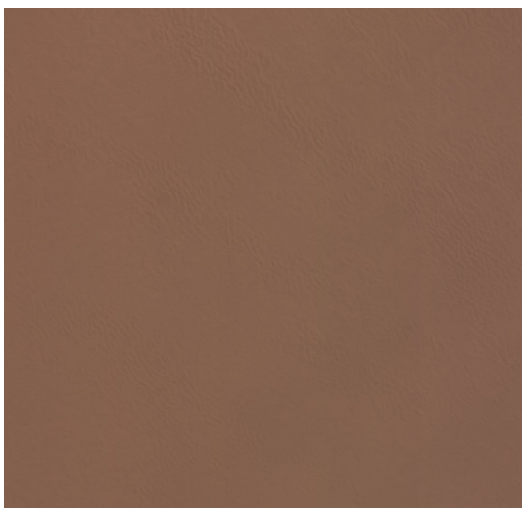

Nigeria_2

Figure 3. PLEIADES acquisitions of the six African desert sites used for its absolute calibration (coloured composition of the multispectral bands Blue, Green and Red).

These sites are very complementary because of the spectral behaviour of their surface and also of the atmospheric properties of the environment. Figure 2 presents the spectral curves of these 5 sites from the blue to the near infrared wavelength. The intensity and the slopes of the surface (water, sand, snow, vegetation and bare soil for $\mathrm{La} C \mathrm{Crau}$, and the Moon) are very different. These characteristics are very crucial in order to characterize or just take into account the spectral variability of the instrument along its field of view.

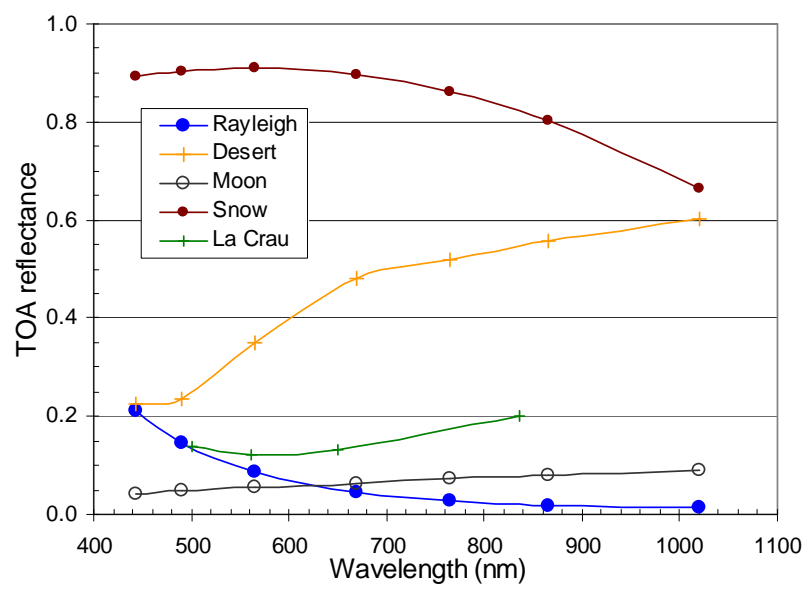

Figure 2. TOA reflectance of the different calibration sites.

\subsection{Desert sites}

Twenty African desert sites are usually used by the CNES to inter-calibrate sensors. The size of these standard sites are $0.45^{\circ} \mathrm{x} 0.45^{\circ}$. Its size is well adapted to sensors with a large swath as PARASOL, MODIS or MERIS but not adapted to sensors with a very high resolution such as PLEIADES or VEN $\mu$ S. Some "small" sites have then be derived from the standard ones in order to be compliant for sensors with a small swath. This small sites are characterized by a size of $0.2^{\circ} \times 0.2^{\circ}$. Their position inside the standard site has been chosen in order to guarantee the homogeneity of these small sites, their temporal stability and also radiometric properties very closed to the standard one allowing inter-calibration between a sensor with a wide swath with a sensor characterised by a narrow swath. Among the twenty desert sites, six of the most homogeneous sites have been selected for the PLEIADES absolute calibration: Algeria_3, Algeria_5, Arabia_1, Libya_1, Niger_2 and Mali_1. Table 1 shows the position of their centre in latitude and longitude whereas Figure 3 shows PLEIADES acquisitions of theses six sites.

\begin{tabular}{|l|cc|}
\hline Small Sites & Latitude & Longitude \\
\hline Algeria_3 & 30,32 & 07,66 \\
Algeria5 & 31,02 & 02,23 \\
Arabia_1 & 18,88 & 46,76 \\
Libya_1 & 24.50 & 13.30 \\
Mali_1 & 19,12 & $-04,85$ \\
Nigeria_2 & 21,37 & 10,59 \\
\hline
\end{tabular}

Table 1 : Position of the centre of the small sites. 


\subsection{Antarctic sites}

The four sites located in the Antarctic, Dome_1, Dome_2, Dome_ 3 and Dome_C have also been used for the calibration of PLEIADES. PLEIADES' acquisitions were centred in the middle of these sites. Acquisitions are only possible between December and February when the sun is high enough to allow radiative transfer simulations.

\begin{tabular}{|c|c|c|}
\hline & Latitude & Longitude \\
\hline Dôme 1 & $-78.5^{\circ}$ & $120^{\circ}$ \\
\hline Dôme 2 & $-75.7^{\circ}$ & $113^{\circ}$ \\
\hline Dôme 3 & $-77.38^{\circ}$ & $128.71^{\circ}$ \\
\hline Dôme C & $-75.1^{\circ}$ & $123^{\circ}$ \\
\hline
\end{tabular}

Table 2: Position of the centre of the Antarctic sites.

These sites are characterised by a specular effect due to the snow and also due to the sastrugis (small relief of snow/ice created by the wind) (Hudson, 2006). The high resolution of PLEIADES, $70 \mathrm{~cm}$ at nadir for the panchromatic band, offers precious information about these structures in order to better understand the bidirectional behaviour of the sites and also the potential differences between the four calibration sites. Figure 4 illustrates the different structures of the sites. The periodicity of these dunes is about $20 \mathrm{~m}$.

\subsection{Ocean sites}

Oceanic sites are also good candidates for calibration because of their behaviour in terms of spatial homogeneity and temporal stability. (Fougnie, 2002) shows that the major oligotrophic

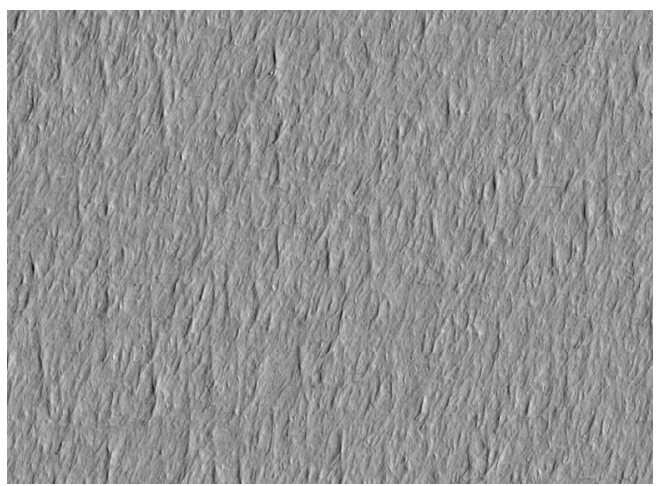

Dome_1

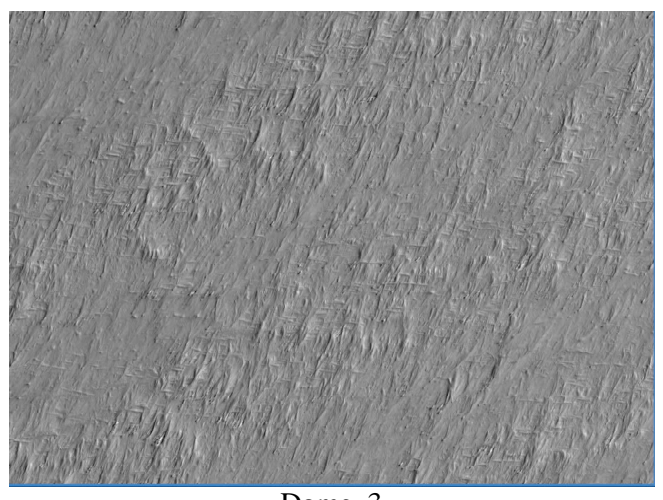

Dome_3 sites are located in North/South Atlantic and Pacific oceans and in Indian ocean (Table 3).

\begin{tabular}{|c|c|c|c|c|}
\hline Localisation & $\begin{array}{c}\text { Latitude } \\
\text { min }\end{array}$ & $\begin{array}{c}\text { Latitude } \\
\text { max }\end{array}$ & $\begin{array}{c}\text { Longitude } \\
\text { min }\end{array}$ & $\begin{array}{c}\text { Longitude } \\
\text { max }\end{array}$ \\
\hline $\begin{array}{c}\text { South-East } \\
\text { Pacific }\end{array}$ & -44.9 & -20.7 & -130.2 & -89.0 \\
\hline $\begin{array}{c}\text { North-West } \\
\text { Pacific }\end{array}$ & 10.0 & 22.7 & 139.5 & 165.6 \\
\hline $\begin{array}{c}\text { North } \\
\text { Pacific }\end{array}$ & 15.0 & 23.5 & 179.4 & 200.6 \\
\hline $\begin{array}{c}\text { North } \\
\text { Atlantic }\end{array}$ & 17.0 & 27.0 & -62.5 & -44.2 \\
\hline $\begin{array}{c}\text { South } \\
\text { Atlantic }\end{array}$ & -19.9 & -9.9 & -32.3 & -11.0 \\
\hline South India & -29.9 & -21.2 & 89.5 & 100.0 \\
\hline
\end{tabular}

Table 3: Position of the ocean calibration sites.

PLEIADES' acquisitions are programmed over these sites in backscattering conditions in order to avoid the glitter. As it is very difficult to obtain clear sky conditions over these areas, the narrow swath of PLEIADES $(20 \mathrm{~km})$ is compensated by a large track (about $100 \mathrm{~km}$ ).

\subsection{La Crau site}

The La Crau site is located in the south East of France, near Marseille. It is an automatic ground based station named

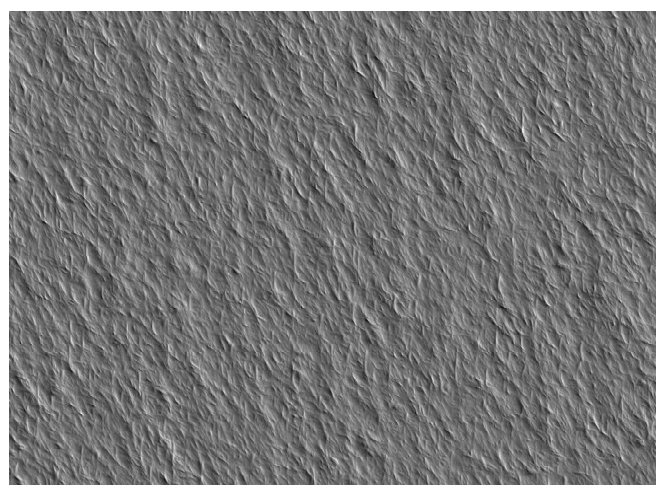

Dome_2

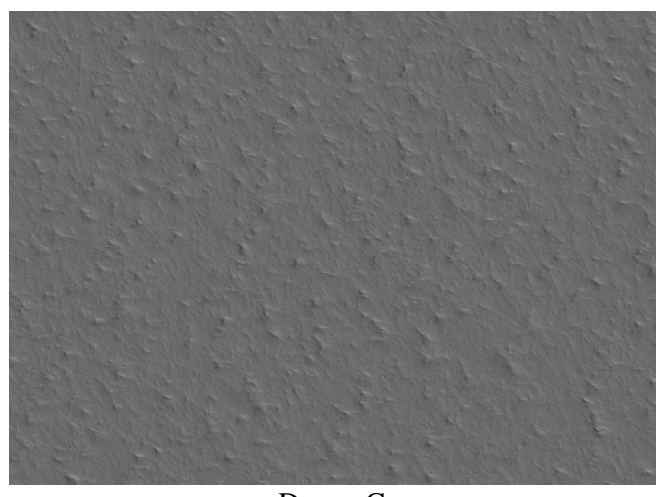

Dome_C

Figure 4 : PLEIADES panchromatic acquisitions of the Dome sites (spatial resolution : $70 \mathrm{~cm}$ ). 
ROSAS (Robotic Station for Atmosphere and Surface), which continuously provides atmosphere and ground characterization (Figure 5).

This system allows us to calibrate any high resolution sensor when it passes over the site. It is composed of 9 filters (from $380 \mathrm{~nm}$ to $1600 \mathrm{~nm}$ ) and 2 collimators equipped with 2 detectors: Si et InGaAs.

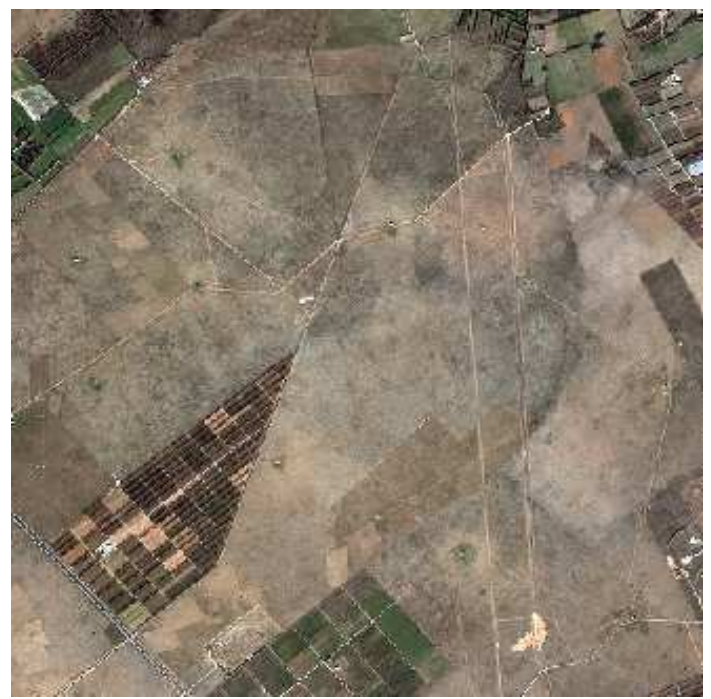

Figure 5: La Crau acquired by PLEIADES the 01/24/12.

\subsection{The Moon}

The moon is the last natural site used as an operational site for the PLEIADES radiometric calibration. In a nominal calibration mode, the moon is observed once a month during the descending phase in order to follow the stability of the absolute calibration of the different spectral bands.

The image below shows a lunar acquisition of PLEIADES with a phase of about $-40^{\circ}$.

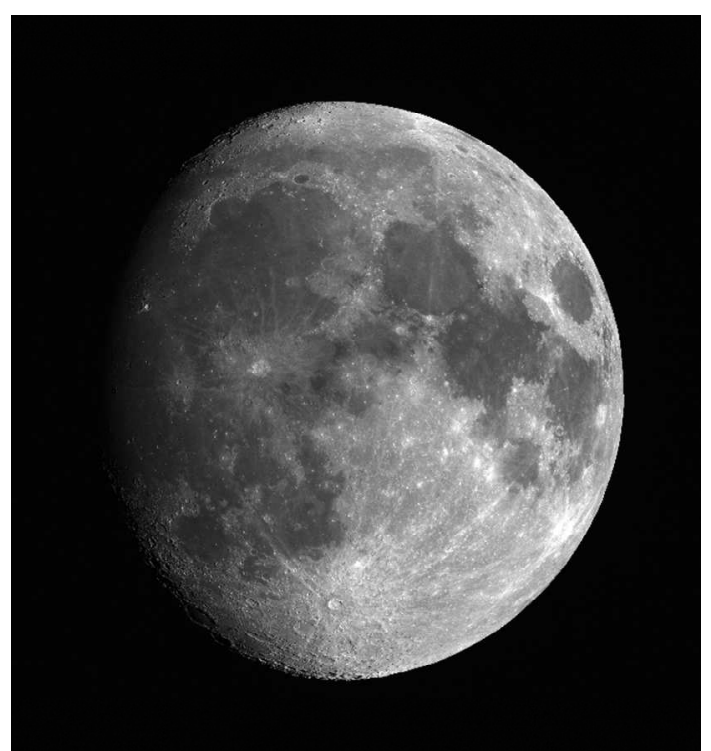

Figure 5: Acquisition of the moon, acquired by PLEIADES the 02/04/12.

\section{CALIBRATION METHODS}

Different calibration methods, based on the previous sites, are used to assess the absolute calibration coefficients of the five spectral bands of PLEIADES: absolute calibration methods, cross-calibration ones, multi-temporal calibration methods and inter-band calibration methods. These methods are already described in detail in other papers, so this article will just highlight the way to combine them in order to calibrate a new sensor.

\subsection{Absolute calibration methods}

Two sites could be used in order to provide directly absolute calibration coefficients: La Crau and Oceans.

As explained previously, an automatic station is settled in the site of La Crau and provides automatically information allowing us to calculate the calibration coefficients (Meygret, 2005). This information is: aerosol optical depth in the different spectral bands of the station, water vapour content and a bidirectional characterization of the ground reflectance. This site could thus be used to simulate the radiance measured by the sensors and thus to derive absolute calibration coefficients for sensors with a high spatial resolution like PLEIADES.

The second natural site which directly provides an absolute calibration is the oceans. Indeed, the top-of-atmosphere (TOA) signal measured by a satellite sensor observing oceanic targets is in large proportion due to the scattering of the incident solar irradiance by atmospheric components, especially in the visible range. In this spectral range, the molecular scattering, so-called Rayleigh scattering, is the main process contributing to the TOA signal and this contribution can be accurately predicted and computed using surface pressure, knowing the spectral response of the instrument. Other processes contributing to the TOA signal are aerosol scattering, back scattering by the water body, diffuse reflection by whitecaps, specular (or Fresnel) reflection by the surface, and gaseous absorption.

Satellite acquisitions over such oceanic targets can be selected so that the contribution of these secondary processes is minimized. For these acquisitions, the molecular scattering signal may constitute as much as $90 \%$ of the TOA signal, for spectral bands from blue to red bands (typically 443 to $670 \mathrm{~nm}$ ). This forms the basis of the calibration method using Rayleigh scattering (Fougnie, 2007).

\subsection{Cross calibration methods}

In order to verify the results obtained over the La Crau sites for all the spectral bands and over the oceans for the spectral bands from the blue to the red, cross calibration methods are also performed over desert and Antarctic sites.

The principle of a cross-calibration approach is to compare the radiance (or reflectance) measured by the sensor to calibrate, with a reference radiance, computed using radiances measured by another sensor, called the reference sensor (Lachérade, 2012).

In the case of PLEIADES, three different sensors were chosen to perform this comparison: PARASOL, MERIS and MODIS. These sensors were chosen because of the great coverage of their spectral bands which allow us to derive the reflectance of PLEIADES from the reflectance measured in their own spectral bands.

In order to take into account the bidirectional behavior of the sites (backscattering effects for deserts and specular effects for Antarctic), pairs of measurements which are considered are those which were acquired in the same geometrical and solar conditions. The size of this geometrical window, defined with the Equations (1), is a compromise between the need to obtain a sufficient number of matched measurements and the need to 
avoid errors in the inter-calibration due to the bidirectional behaviour of the reflectance of the site.

$$
\begin{gathered}
\left|\theta_{s}^{C A L}-\theta_{s}^{R E F}\right|<2^{\circ} \\
\left|\theta_{v}^{C A L}-\theta_{v}^{R E F}\right|<2^{\circ} \\
|| \varphi_{s}^{C A L}-\varphi_{v}^{C A L}|-| \varphi_{s}^{R E F}-\varphi_{v}^{R E F} \mid<5^{\circ}
\end{gathered}
$$

where

$$
\begin{aligned}
& \left(\theta_{v}, \varphi_{v}\right) \text { are viewing zenith and azimuth angles } \\
& \left(\theta_{s}, \varphi_{s}\right) \text { are solar zenith and azimuth angles }
\end{aligned}
$$

For aerosol optical properties, in the absence of a reliable information source above deserts and Antarctic, a constant representative value of 0.2 (at $550 \mathrm{~nm}$ ), respectively 0.05 for Antarctic, is considered for optical thickness, associated with a desert aerosol model, respectively a continental model for Antarctic. The method relies on statistics to cancel errors due to these assumptions.

\subsection{Multi-temporal calibration methods}

As desert sites have been chosen for their temporal stability, they also could be used to follow the spectral sensitivity of spectral bands from the blue to the short-wave infrared during the lifetime of the sensors. Gamet et al. (2011) carry out the advantages of using desert sites for multi-temporal calibration.

Another very stable site for multi-temporal calibration is the Moon. Indeed, the Moon is without any disturbing meteorological atmosphere such as aerosols. So it allows us to have a very good repetitivity of measurements when geometrical conditions are the same. The approach used to realize this multi-temporal calibration is based on the measurement of the whole surface of the moon. The USGS (United States Geological Survey) has supplied a semiempirical model of the albedo of the Moon named ROLO (RObotic Lunar Observatory). This model takes into account the vibration of the Moon and is able to simulate the global reflectance of the Moon at any date (Stone, 2003). For each lunar acquisition, the calibration method consists in calculating a standardized lunar irradiance integrated on the spectral range of the sensor. This value is in theory invariant and only depends on the sensitivity of the instrument.

To minimize the errors that could occur due to the model, the moon is acquired each month with a descending phase of about $40^{\circ}$.

This method only provides a multi-temporal calibration of the sensor. To obtain an absolute calibration, the first points obtained with this method are fitted "in absolute" on the absolute calibration results obtained with the other methods.

\section{USE OF THE HIGH LEVEL OF AGILITY OF PLEIADES}

PLEIADES is a satellite which is extremely agile. This section highlights three examples of some very interesting acquisitions which have been taken and will be processed in the next few months using its agility:

- the capacity to realize measurements quasisimultaneously;

- the capacity to capture stars with a very good precision;

- the capacity to capture the moon twice a day every day during a lunar cycle.

\subsection{Bidirectional characterization of the surfaces}

Figure 6 is an example of what PLEIADES is able to realize over one site: 23 quasi-simultaneous images in about 4 minutes with yaw angles varying from $-49^{\circ}$ to $+49^{\circ}$. This fantastic agility, added to its high resolution, provides us with the opportunity to characterize the bidirectional reflectance of our calibration sites.

The case illustrated in Figure 6 is on the La Crau site. The analysis of these type of acquisitions will allow us to compare directly the BRDF obtained "on-board" with the BRDF measured on ground by the photometer ROSAS.

This "VIDEO" mode will also help us to understand the bidirectional behaviour of the desert and Antarctic sites and the impact of the sensor's resolution of this behaviour. Figure 7 shows the bidirectional reflectance which has been retrieved on Dome_C after inversion of the Top Of Atsmophere reflectance of PARASOL over the site Dome_C.
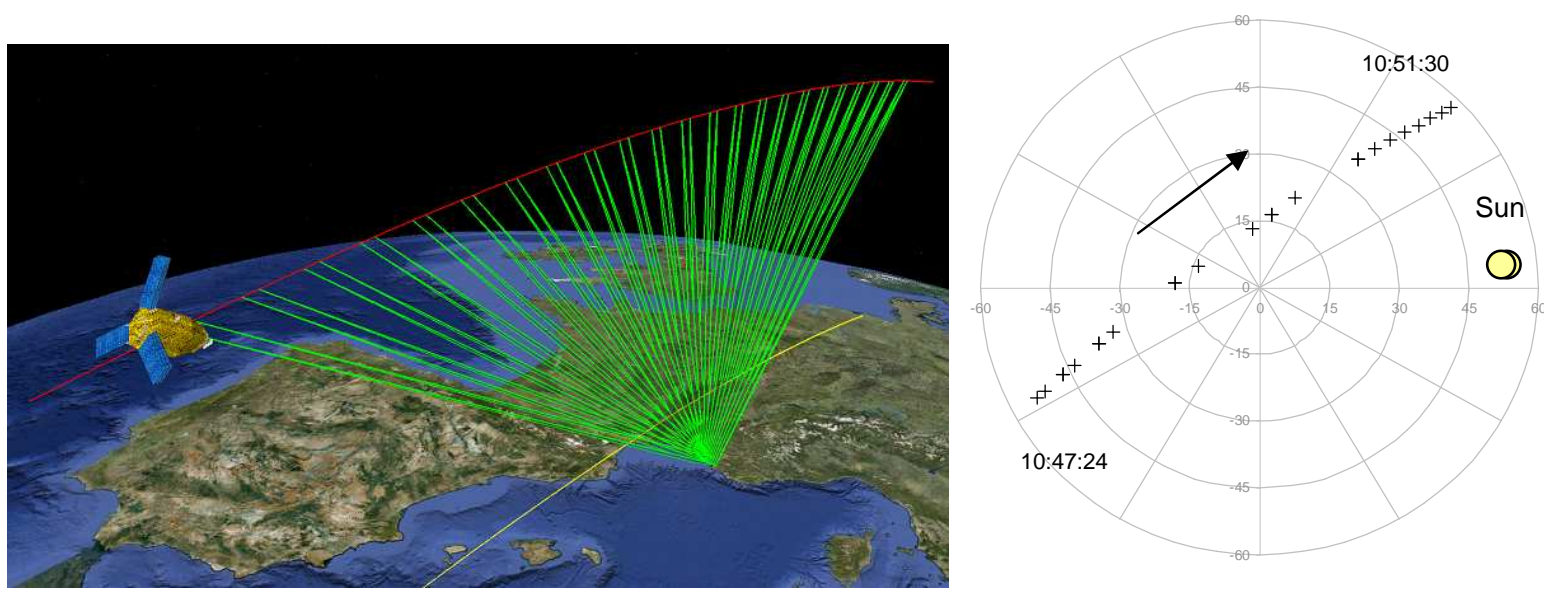

Figure 6 : Acquisitions of 23 quasi-simultaneously images over La Crau

(Left: satellite orbit and acquisitions ; right: position of the acquisitions in a polar graph, relative to the solar principal plane). 
PARASOL is a sensor which has a very large swath and a low resolution (about $1 \mathrm{~km}$ ). The comparison between this result and the one which will be obtained using the high resolution data provided by PLEIADES will be very fruitful.

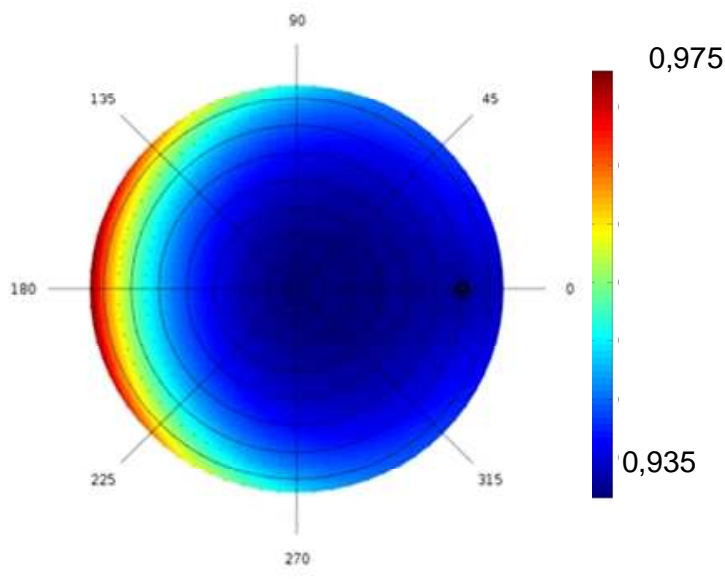

Figure 7 : On ground BRDF processed from PARASOL data on Dome_C.

This technology could also be very useful for the Rayleigh calibration: Do the absolute calibration coefficients depend on the observation viewing angle ? What is the response of the uncertainties on the atmospheric parameters to the viewing angle ? Video acquisitions have been performed over oceans during the first four months of the commissioning phase of PLEIADES. Results will be analysed in the next few months.

\subsection{Absolute calibration using stars}

The last two sections of this article highlight the benefits which can be obtained by using extra-terrestrial acquisitions for absolute calibration (Fourest, 2012).

Stars are very stable elements in the sky and knowing their magnitude and their temperature, it is possible to derive absolute calibration coefficients.

During the commissioning phase, the PLEIADES constellation has been acquired every week in order to follow the temporal evolution of the absolute calibration of the sensor PLEIADES. The results will be compared with those obtained over desert sites and over the Moon.

\subsection{Lunar multi-temporal sensitivity to the phase}

When the Moon is used to perform multi-temporal calibration activities, we completely depend on the ROLO model in order to normalize the successive acquisitions taking into account the differences in phase and librations. An experiment was carried out using the extreme agility of PLEIADES to capture the Moon twice a day with about 1 hour between two acquisitions and this, during several days in order to cover the descending phase from $-110^{\circ}$ to the ascending phase of $+110^{\circ}$, including the full moon. This experiment was conducted twice in order to be able to reproduce the results and to evaluate the precision of this experiment.

The results of this exercise on the calibration results is theoretically a constant line if the calibration sensor is stable (except out of the range of the model which is about $90^{\circ}$ ). Another result of this experiment will be the analysis of the behaviour of the model in its extrapolation range.
All of these results will give us important information in order to improve the precision of this calibration method, based on the use of the Moon.

\section{REFERENCES}

Blanchet G., L. Lebegue, S. Fourest, C. Latry, F. Porez-Nadal, S. Lachérade, C. Thiebaut, 2012, Pleiades-HR innovative techniques for Radiometric Image Quality Commissioning," ISPRS Melbourne.

Fougnie B., P. Henry, A. Morel, D. Antoine, and F. Montagner, 2002, Identification and Characterization of Stable Homogeneous Oceanic Zones : Climatology and Impact on Inflight Calibration of Space Sensor over Rayleigh Scattering," Proceedings of Ocean Optics XVI, Santa Fe, New Mexico.

Fougnie B., G. Bracco, B. Lafrance, C. Ruffel, O. Hagolle, and C. Tinel, 2007, PARASOL In-flight Calibration and Performance, Applied Optics, 46(22), pp. 5435-5451.

Fourest S., L. Lebegue, C. Dechoz, S. Lachérade, G. Blanchet, 2012, Star-based methods FOR Pleiades HR commisioning, ISPRS Melbourne.

Gamet P., S. Lachérade, B. Fougnie, C. Thomas, 2011, Calibration of VIS/NIR sensors over desert sites : New results for cross- and multi-temporal calibration, Conference on Characterization and Radiometric Calibration for Remote Sensing, Logan, USA.

Hudson S.R., S. G. Warren, R. E. Brandt, T. C. Grenfell, D. Six, 2006, Spectral bidirectional reflectance of Antarctic snow: Measurements and parameterization, Journal of Geophysical research, vol. 111, D18106.

Lachérade S., Cross-Calibration Over Desert Sites : Description, Methodology and Operational Implementation, IEEE Transactions on Geoscience and Remote Sensing, InterCalibration of Satellite Instruments Special Issue, Submitted in January 2012.

Meygret A. 2005, Absolute Calibration: from SPOT1 to SPOT5, SPIE, San Diego.

Stone T. C., H. H. Kieffer, and K. J. Becker, 2003, Modeling the Radiance of the Moon for On-orbit Calibration, Proc. SPIE 5151, 463-470.

\section{ACKNOWLEDGEMENTS}

The author would like to thank the whole team of image experts who have performed a great job, spending time, energy and enthusiasm during the 6 months of the image quality commissioning. She would also like to thank the Pleiades image ground processing team for its support and especially the ICC (Image Calibration Centre) exploitation and Pleiades Mission teams for their constant good mood, providing the experts with acquisitions and image products. 